\title{
Gallbladder Fistula Treated with N-Butyl-2-Cyanoacrylate after Radiofre- quency Ablation in a Hepatocellular Carcinoma Patient: a Case Report
}

\author{
In Tae So', Byoung Kook Jang', Jae Seok Hwang', Young hwan Kim² \\ 'Department of Internal Medicine, Keimyung University School of Medicine; ${ }^{2}$ Department of Radiology, Daegu Catholic University Medical \\ Center, Catholic University of Daegu College of Medicine, Daegu, Korea
}

Received Jan. 27, 2019

Revised Feb. 11, 2019

Accepted Feb. 11, 2019
Radiofrequency ablation (RFA) is a minimally invasive local therapy for hepatocellular carcinoma (HCC). Even though RFA is considered to be a safe treatment modality, a variety of complications have been reported. Recently, we encountered a case of refractory fistula between a liver abscess and the gallbladder after RFA. A 64-year-old woman diagnosed with HCC associated with chronic hepatitis B was treated by RFA. After RFA, she experienced abdominal pain, and abdominal computed tomography (CT) revealed a liver abscess complicated by a previous treatment of HCC, she was treated with intravenous antibiotics and percutaneous abscess drainage. Follow-up abdominal CT revealed a fistula between the liver abscess and gallbladder, which was successfully treated with percutaneous transcatheter n-butyl-2-cyanoacrylate (NBCA) embolization. We herein report the rare case of a refractory fistula between a liver abscess and the gallbladder after RFA in a patient treated with NBCA embolization. (J Liver Cancer 2019;19:69-73)

Keywords: Hepatocellular carcinoma; Radiofrequency ablation; Fistula; N-butyl-2-cyanoacrylate

\section{INTRODUCTION}

Although the mainstay of hepatocellular carcinoma (HCC) therapy is surgical resection, the majority of patients are not treated by surgery because of tumor extent and poor hepatic reserve. ${ }^{1}$ Radiofrequency ablation (RFA) is a minimally invasive local therapy that is a thermal ablation technique for achieving coagulative necrosis of HCC and has been considered a relatively safe treatment modality. ${ }^{2,3}$ However, RFA may cause complications, such as injury to the adjacent normal tissue related to mechanical and thermal damages. We

\footnotetext{
Corresponding author: Jae Seok Hwang

Department of Internal Medicine, Dongsan Medical Center, Keimyung University School of Medicine, 56 Dalseong-ro, Jung-gu, Daegu 41931, Korea Tel. +82-53-250-7088, Fax. +82-53-250-7442

E-mail; gastro@dsmc.or.kr

https://orcid.org/0000-0002-1510-3534
}

report the case of a refractory fistula that developed between a liver abscess and the gallbladder after RFA in a patient that was successfully treated with percutaneous transcatheter nbutyl-2-cyanoacrylate (NBCA) embolization.

\section{CASE REPORT}

A 64-year-old female patient presented to the hospital with the chief complaint of right upper quadrant abdominal pain. Five years prior, she had been diagnosed with HCC associated with chronic hepatitis B and had undergone repeated transarterial chemoembolization. Ten days prior, the patient was discharged without complications after RFA due to HCC recurrence in segment 8 . She had no alcohol consumption and smoking history. With the exception of appendectomy and vitiligo, she had no medical history. Physical examination showed tenderness in the right upper abdomen. She ap- 
peared acutely ill, with a blood pressure of $74 / 40 \mathrm{mmHg}$, heart rate of 84 beats/min, respiratory rate of 18 breaths $/ \mathrm{min}$, and body temperature of $37.5^{\circ} \mathrm{C}$.

Laboratory test results showed a white blood cell count of $35,420 / \mathrm{mm}^{3}$, hemoglobin level of $11.9 \mathrm{~g} / \mathrm{dL}$, platelet count of $432,000 / \mu \mathrm{L}$, aspartate aminotransferase level of $31 \mathrm{IU} / \mathrm{L}$, alanine aminotransferase level of $24 \mathrm{IU} / \mathrm{L}$, total protein level of $6.8 \mathrm{~g} / \mathrm{dL}$, total albumin level of $4.1 \mathrm{~g} / \mathrm{dL}$, prothrombin time of 14.4 seconds, total bilirubin concentration of $0.5 \mathrm{mg} / \mathrm{dL}$, alpha-fetoprotein level of $33.2 \mathrm{ng} / \mathrm{mL}$, lactate level of 6.1 $\mathrm{mmol} / \mathrm{L}$, and C reactive protein level of $11.22 \mathrm{mg} / \mathrm{dL}$. Hepatitis virus markers showed hepatitis $\mathrm{B}$ surface antigen positivity, hepatitis B surface antibody negativity, immunoglobulin G anti-hepatitis B core antigen positivity, serum hepatitis B virus DNA level $<15 \mathrm{IU} / \mathrm{mL}$, and anti-hepatitis $\mathrm{C}$ virus negativity.

Dynamic abdominal computed tomography (CT) that was conducted at the time of the hospital visit showed complete ablation of the HCC in segment 8 , but also a low-density mass with irregular margins suggestive of a liver abscess at the RFA site and perihepatic fluid collection (Fig. 1).

The patient was diagnosed with liver abscess and septic shock after RFA in consideration of hypotension, fever, and

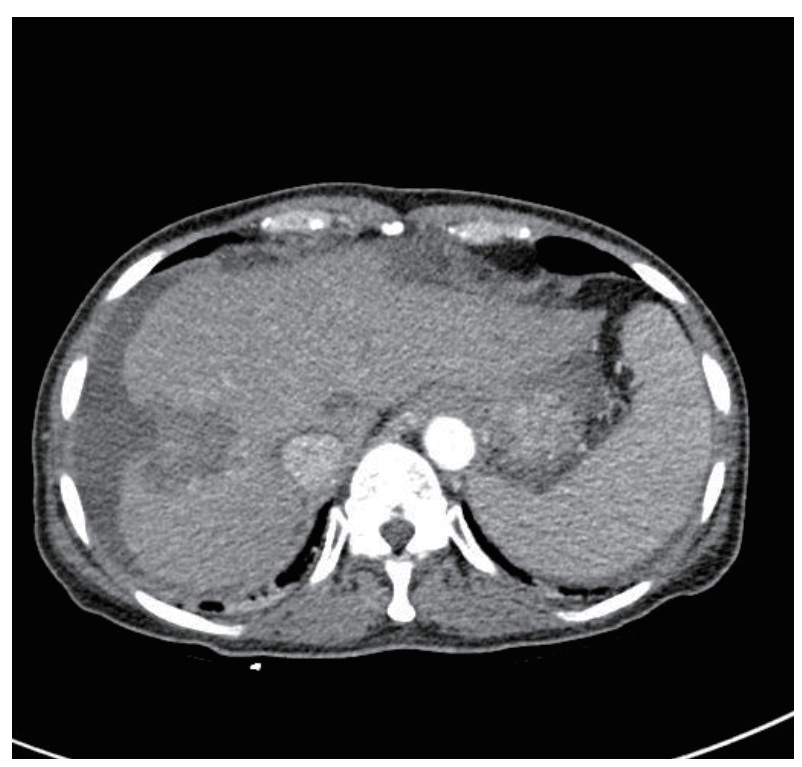

Figure 1. Dynamic CT during arterial phase showing an ill-defined, irregularly shaped liver abscess in the right anterior, superior segment of the liver and perihepatic fluid collection. $\mathrm{CT}$, computed tomography. laboratory and CT findings. She was hospitalized, was administered intravenous empirical antibiotics and inotropics, and underwent ultrasound-guided percutaneous abscess drainage by placement of a pigtail catheter in the abscess. The patient's vital signs were stabilized, and abdominal pain and leukocytosis also improved. While the drainage amount gradually decreased, from the 14 days of pigtail catheter insertion, 50-80 mL of greenish fluid was continuously drained. Drained fluid analysis showed a greenish color and polydominant exudate suggestive of a bile and pus mixture. Followup abdominal CT was performed on the 18th day of pigtail catheter insertion to check for complications, and CT showed suspicion of a liver abscess and gallbladder fistula (Fig. 2). The tubography via the pigtail catheter also revealed a fistulous communication between the gallbladder and liver abscess (Fig. 3). Surgical resection including cholecystectomy was considered for fistula treatment, but the patient refused the surgery because of concern about the associated complications and risk. Therefore, we expected the closure of the fistula while continuing the conservative treatment of percutaneous abscess drainage and antibiotics. However, the fistula that failed to resolve after 4 weeks of conservative treatment was considered persistent, and right pleural effusion

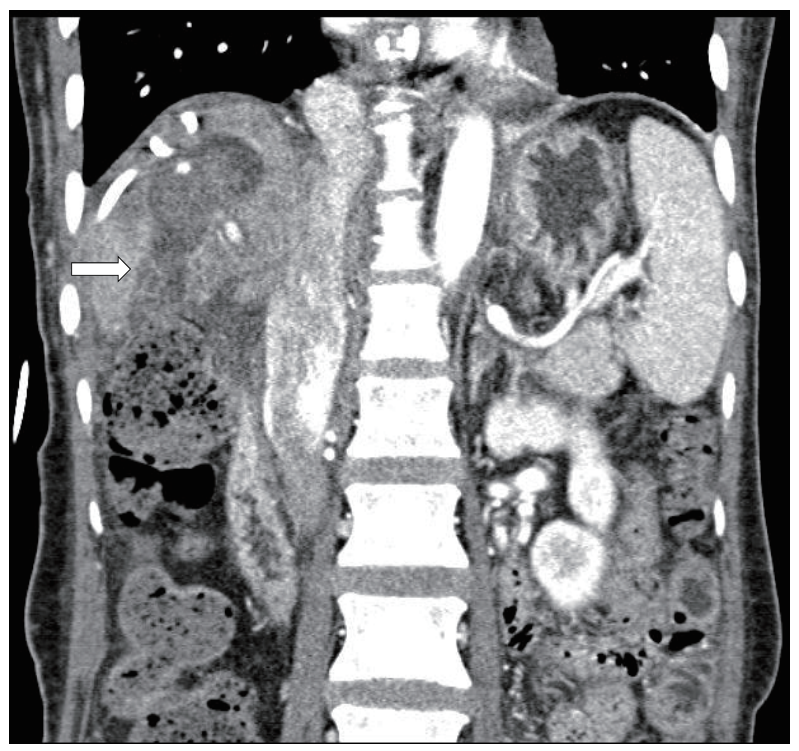

Figure 2. Dynamic CT showing an abscess and gallbladder fistula (white arrow). CT, computed tomography. 
occurred; we decided to perform embolization of the persistent fistula tract using NBCA (Histoacryl ${ }^{\circledR}$, B. Braun Avitum AG, Spangenberg, Germany).

NBCA and oily contrast agent Lipiodol Ultra-Fluid (Guerbert $\mathrm{GmbH}$, Sulzbach, Germany) were mixed in a 1:1 ratio

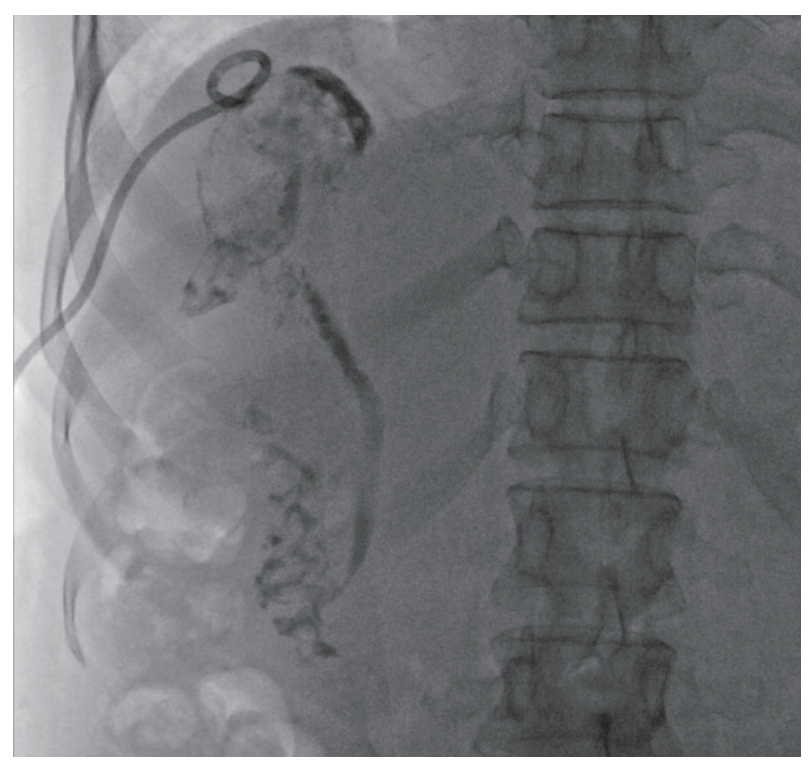

Figure 3. Tubography via a pigtail catheter showing the passage of the contrast dye through the gallbladder, cystic duct, common bile duct, and duodenum due to fistula formation between the abscess and gallbladder. and injected at a volume and rate suitable for polymerization reaction to fill and seal the gallbladder, fistula tract, and remnant abscess cavity (Fig. 4). After the embolization procedure, the percutaneous drainage catheter was withdrawn. Seven days after the procedure, CT showed that the NBCA had sealed the remnant abscess cavity, fistula tract, and gallbladder, and tubography confirmed fistula tract occlusion through non-visualization of the cystic duct, common bile duct, and duodenum (Figs. 5, 6). The patient was discharged and was followed up periodically in the outpatient clinic with abdominal CT. Fistula-related complications no longer occurred, and NBCA and Lipiodol Ultra-Fluid mixtures were no longer visible on follow-up CT 2 years later.

\section{DISCUSSION}

RFA is widely used as a curative treatment modality for early HCC, which has excellent treatment outcomes and low risks for procedures. The incidence rates of complications were found to be $2.4 \%$ in domestic studies and $1.5 \%$ in other studies. ${ }^{4,5}$ Complications include mild complications, such as fever, abdominal pain, and general weakness, which spontaneously subside over time, and serious major complications,
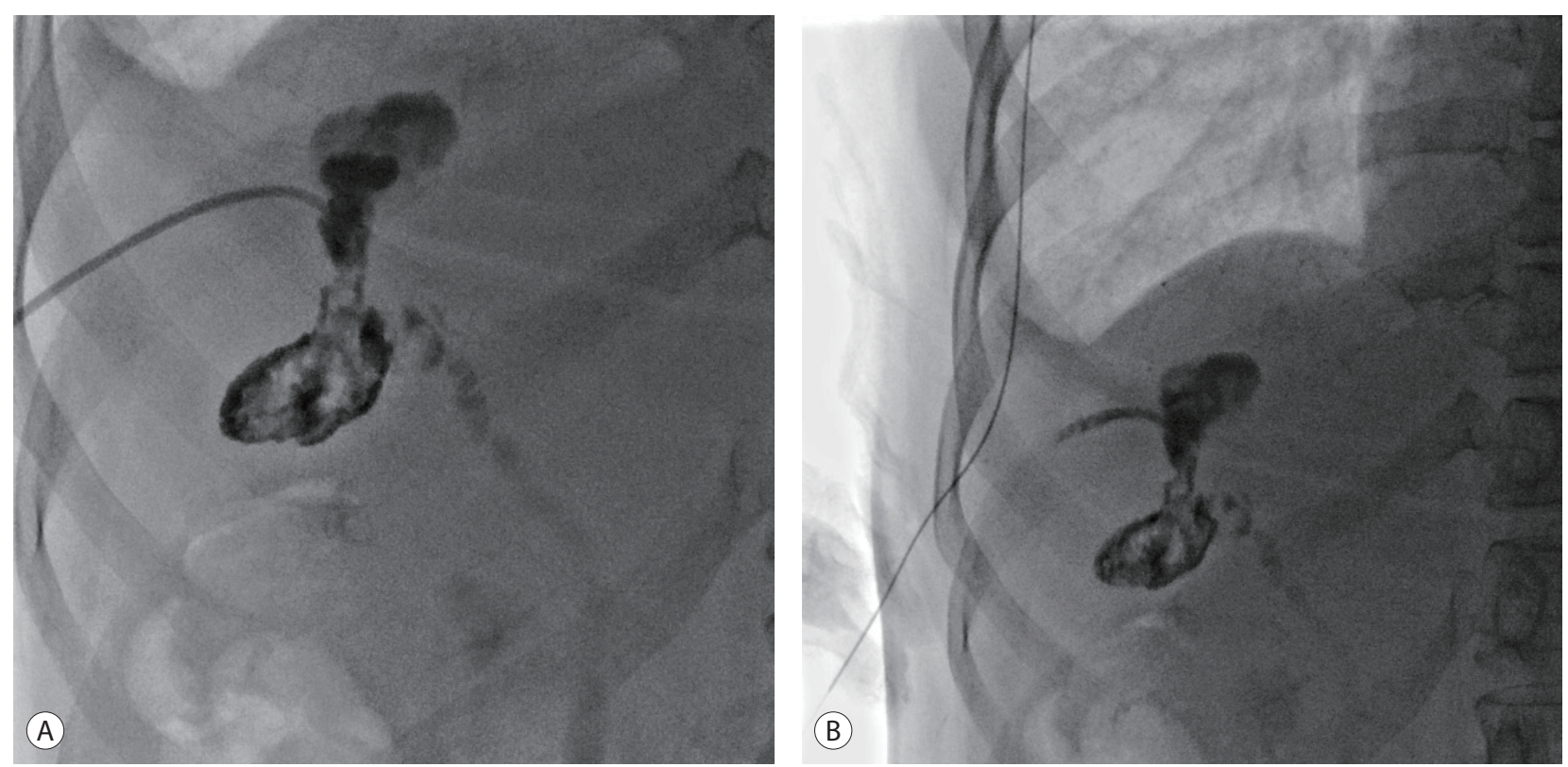

Figure 4. (A) Tubography showing fistula embolization with an NBCA-lipiodol mixture, and NBCA is observed along the previous remnant abscess, fistula tract, and gallbladder. (B) After the fistula tract embolization procedure, the percutaneous drainage catheter was removed. NBCA, n-butyl-2-cyanoacrylate. 


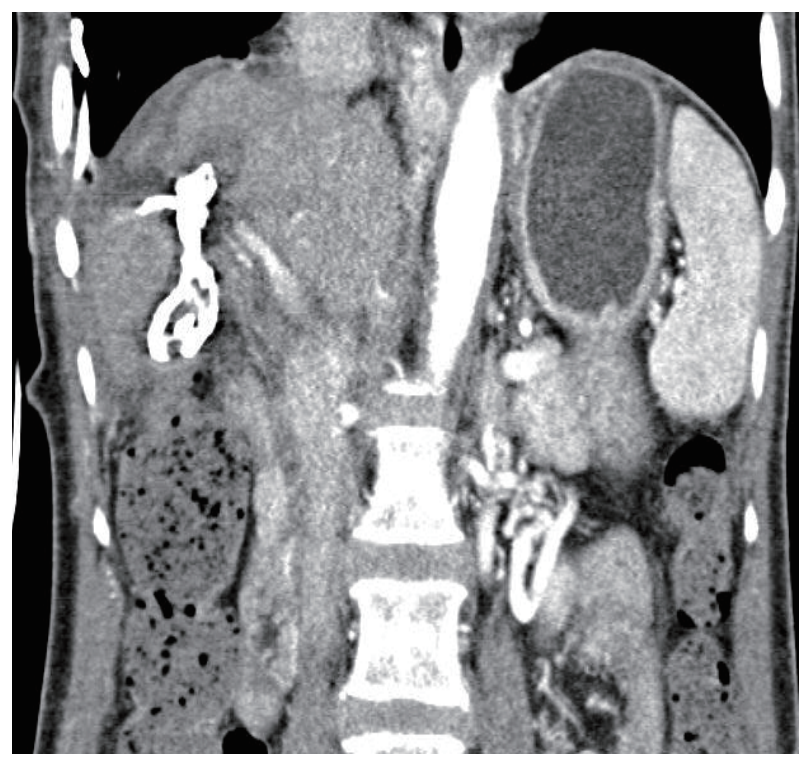

Figure 5. CT showing the remnant abscess, fistula tract, and gallbladder cavity sealed with NBCA. CT, computed tomography; NBCA, n-butyl-2-cyanoacrylate.

which may lead to death if they are not adequately diagnosed and treated, such as liver abscess, intraabdominal hemorrhage, hepatic infarction, bowel perforation, tumor seeding, biliary duodenal fistula, and abscess-colonic fistula. ${ }^{4-6}$ The incidence rates of major complications in both domestic and other multicenter studies were found to be $2.4 \%$ and $4.0 \%$, respectively. ${ }^{5,6}$ Among major complications, liver abscess is the most common. ${ }^{5}$ Image-guided percutaneous drainage is the mainstay therapy, but this therapy modality often has limited effectiveness in the presence of an associated fistula. There is no standard treatment for a liver abscess with a fistula. When only an abscess is treated without the fistula resolution, it causes recurrent infection or secondary complications. Our patient refused surgical treatment for fistula that did not respond to antibiotics and percutaneous drainage therapy. Therefore, nonsurgical treatment was considered, and the fistula was treated using NBCA, which is used to embolize the vascular lumen and fistula of extravascular structures. Percutaneous treatments of non-healing fistulas with the use of NBCA have been reported in patients with colonic-abscess, biliary-enteric, tracheoesophageal, and bronchopleural fistulae. .10 $^{7-10}$

This is the first case report of a fistula between a liver ab-

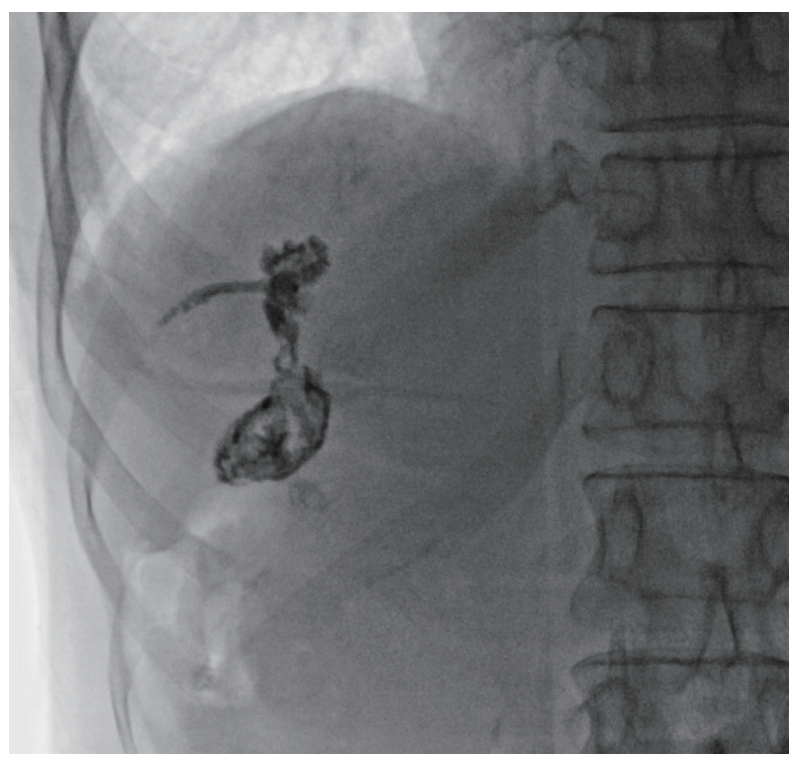

Figure 6. Tubography showing the fistula tract occlusion through non-visualization of the cystic duct, common bile duct, and duodenum after dye injection.

scess and the gallbladder after RFA that was treated using percutaneous transcatheter NBCA embolization. Surgical resection may be considered for the treatment of a fistula, but the surgical risk is high when considering the patients' reduced liver function and performance status due to underlying cirrhosis and HCC. This case suggests that percutaneous NBCA embolization may be a therapeutic option for persistent fistula between a liver abscess and the gallbladder with an alternative to major surgical intervention. However, there is no large-scale study of the results of treatment including delayed complications and recurrence after percutaneous radiologic intervention using NBCA embolization in a fistula, so further studies are needed.

\section{Conflicts of Interest}

The authors declare no conflicts of interest relevant to this article.

\section{REFERENCES}

1. Bruix J, Llovet JM. Prognostic prediction and treatment strategy in hepatocellular carcinoma. Hepatology 2002;35:519-524.

2. Rossi S, Buscarini E, Garbagnati F, Di Stasi M, Quaretti P, Rago M, et 
al. Percutaneous treatment of small hepatic tumors by an expandable RF needle electrode. AJR Am J Roentgenol 1998;170:10151022.

3. Bai XM, Yang $W$, Zhang ZY, Jiang AN, Wu W, Lee JC, et al. Longterm outcomes and prognostic analysis of percutaneous radiofrequency ablation in liver metastasis from breast cancer. Int J Hyperthermia 2019;35:183-193.

4. Livraghi T, Solbiati L, Meloni MF, Gazelle GS, Halpern EF, Goldberg $\mathrm{SN}$. Treatment of focal liver tumors with percutaneous radio-frequency ablation: complications encountered in a multicenter study. Radiology 2003;226:441-451.

5. Rhim H, Yoon KH, Lee JM, Cho Y, Cho JS, Kim SH, et al. Major complications after radio-frequency thermal ablation of hepatic tumors: spectrum of imaging findings. Radiographics 2003;23:123-134; discussion 134-136.
6. Bertot LC, Sato M, Tateishi R, Yoshida H, Koike K. Mortality and complication rates of percutaneous ablative techniques for the treatment of liver tumors: a systematic review. Eur Radiol 2011;21:2584-2596.

7. Bae JH, Kim GC, Ryeom HK, Jang YJ. Percutaneous embolization of persistent biliary and enteric fistulas with Histoacryl. J Vasc Interv Radiol 2011;22:879-883.

8. York JA. Treating bronchopleural fistulae percutaneously with Nbutyl cyanoacrylate glue. J Vasc Interv Radiol 2013;24:1581-1583.

9. Nunes TF, de Santi GF. Percutaneous embolization of a colonic fistula using n-butyl-2 cyanoacrylate (Histoacryl[R]). Tech Coloproctol 2017;21:763-765.

10. Lopes MF, Catré D, Reis A, Leitão LM. Endoscopic treatment of recurrent tracheoesophageal fistula with histoacryl glue. Gastrointest Endosc 2010;72:1324-1325; author reply 1325. 